positive effects of clinical pilates exercises in individuals with FM. In order to increase the effectiveness of treatment in individuals with FM, we recommend the use of connective tissue massage as an additional treatment method. References:

[1] Busch, Angela J., et al. Exercise therapy for fibromyalgia. Current pain and headache reports 2011;15: 358 .

[2] Burckhardt CS. Nonpharmacologic management strategies in fibromyalgia. Rheumatic diseases clinics of North America, 2002, 28(2),291-304.

Disclosure of Interests: None declared

DOI: 10.1136/annrheumdis-2020-eular.6316

\section{AB0955 \\ FEATURES OF THE PAIN SYNDROME IN RHEUMATOID ARTHRITIS (RA)}

S. Lezhenina ${ }^{1}$, N. Shuvalova ${ }^{2}$, A. Lammert ${ }^{2}$, E. Guryanova ${ }^{1} \cdot{ }^{1}$ Chuvash State University, Cheboksary, Russian Federation; ${ }^{2}$ I. Ya. Yakovlev Chuvash Pedagogical State University, Cheboksary, Russian Federation

Background: The neuropathic component is present in the mechanism of pain in RA in $36 \%$ of cases. The presence of anxiety-depressive disorders and a decrease in the quality of life in patients with RA are shown.

Objectives: The study of the clinical features of pain in RA in men and women. Methods: The group consisted of 134 patients with RA ( 94 women and 40 men), aged 36 to 60 years (average age $48.6 \pm 7.1$ years) and disease duration from 1 year to 10 years $(4.03 \pm 1.6$ years $)$ hospitalized in the rheumatology department of the Republican Clinical Hospital (Cheboksary). At the time of inclusion in the study, all patients were in the active stage of the disease.

An assessment of rheumatological and neurological status was carried out. Pain assessment was performed using: Visual Analog Scale (VAS); Ritchie articular index (RAI). The severity of neuropathic pain was determined using the diagnostic neuropathic pain questionnaire DN4 and PainDetect (sensitivity - 82.9\%; specificity - 89.9\%). To determine the psycho-emotional deviations used: general health questionnaire (anxiety and depression) - General Health Questionnaire (form GHQ - 28); HADS; Spilberger-Hanin situational and personal anxiety scale. Patient mobility limitations were assessed using the Rivermead mobility index score scale, and quality of life was quantified using the EQ-5D visual analogue scale.

To assess the activity of the disease, the level of $\mathrm{C}$-reactive protein (CRP), the erythrocyte sedimentation rate (ESR), and the DAS index - 28-CRP were used. To assess mental and physical functioning, a standardized questionnaire The Short Form-36 was used.

To visualize the stage, survey radiographs were used in the direct projection of the metacarpophalangeal and metatarsophalangeal joints, wrist joints, proximal interphalangeal joints of the hands; distal parts of the feet.

Results: An analysis of chronic pain syndrome in $36 \%$ of patients revealed a neuropathic component of pain (DN4: $5.7 \pm 1.1$ points, PainDetect: $16.3 \pm 4.2$ ). In the group of patients with neuropathic pain $(n=78)$ aged $55.1 \pm 7.9$ years, the duration of the disease was $3.4 \pm 0.9$ years, the more advanced and late clinical stages of the disease, III - IV radiological stages of RA were more common, were present neurological disorders and complaints characteristic of peripheral polyneuropathy. Rivermead mobility index in patients with neuropathic disorders, ( $n=78$ ) was $9.1 \pm 0.8$ points, in the absence of neuropathic disorders $(n=56)$, $11.2 \pm 1.1$ points. There were no significant differences in process activity (DAS index - 28 - CRP) and quality of life.

According to the questionnaire of situational and personal anxiety, Spilberger-Khanin revealed moderate anxiety and mild - depressive disorders. Quality of life was reduced in all patients with RA.

Pain syndrome in patients with neuropathic pain with symptomatic (NSAIDs, GC) and basic cytostatic therapy (methotrexate) showed that, despite the decrease in the severity of the inflammatory process, the positive dynamics was partial (VAS before therapy $6.4 \pm 0,7$; VAS in the presence of therapy $4.3 \pm 0.5$ ( $p>0.05$ ); PainDetect $=14.9 \pm 4.4$; DN4 $=4.3 \pm 1.5$ ).

In men, statistically significant factors associated with pain were clinical parameters that accounted for $37 \%$ and $18 \%$ of pain variation (Ritchie articular index (CIR): Fsmc = 4.107, $p<0.001$; SF-36: Fsmc $=2.107, p<0.001$ ) In women, the main significant factors associated with pain were the subjective feeling of pain and psychological characteristics that accounted for $12 \%$ of the pain variation (SF-36: Fsmc $=11.118, p<0.001$ ).

Conclusion: A dynamic study of patients with RA in accordance with gender and age will further develop additional criteria for evaluating the effectiveness of complex therapy used to treat chronic pain, and will also increase the overall effectiveness of treatment.

Disclosure of Interests: None declared

DOI: 10.1136/annrheumdis-2020-eular.6704

\section{AB0956 \\ VERTICAL NAIL RIDGING IN PATIENTS WITH FIBROMYALGIA: FREQUENCY, PROPOSED GRADING AND CORRELATION WITH OTHER DISEASE FEATURES}

A. Moshrif ${ }^{1}$, M. Abdelkareem ${ }^{1}$, M. Moneer ${ }^{1}$, A. Mosallam ${ }^{1}$, A. Ismail ${ }^{1}$, M. Elwan ${ }^{1}$, A. Saad ${ }^{1}$, T. Abdelaziz ${ }^{1}{ }^{1}$ Al Azhar university, Rheumatology, Assiut, Egypt

Background: The vertical nail ridging (VNR) has long been reported to be related to stressful conditions ${ }^{1}$

Objectives: to evaluate the frequency of VNS in FM patients and its relation to other disease parameters depending on a proposed grading.

Methods: VNR has been searched for in 212 FM patients (2016 criteria). The number of fingers, the degree of VNR according to this proposed grading (0: no ridging, 1: ridging only detected by a magnifying lens, 2 : ridging seen by naked eye and 3: ridging that can be seen and felt) and other FM features according to the new and old ACR criteria have been recorded. 80 subjects of those consulting for knee osteoarthritis have been examined for VNR and those found positive were asked about the FM features and examined for tender points. Patients aged $>50$ years and those with psoriasis and fungal infections were excluded.

Results: the mean age of patients was $32.4 \pm 9.9$ (73.6\% were female). The mean disease duration was $5.8 \pm 3.7$, while the means of WPI, SSS and tender points were $9.4 \pm 2.9,7.3 \pm 1.2$ and $14.7 \pm 2.3$ respectively. VNR was found in 209 patients $(98.6 \%)$. Of 80 controls, VNR has been found in 61 subjects, of whom FM has been diagnosed in 32 patients $(52.4 \%)$ by $2016 \mathrm{FM}$ criteria and in $46(75.4 \%)$ by 1990 criteria. The number of fingers with VNR has been found only correlated with the disease duration $(r=0.276, P=0.000)$. The severity of VNR was significantly correlating with fatigue $(P=0.002)$, sleep disturbance $(P=0.001)$, awaking unrefreshed $(P=0.000)$, WPI $(p=0.01)$ and mean tender points $(P=0.02)$. Considering the 2016 criteria as a gold standard, the sensitivity of VNR was $98.37 \%$, the specificity was $9.68 \%$ and the diagnostic accuracy was $82.8 \%$.

Conclusion: vertical nail ridging is a frequent finding and can be considered helpful for diagnosis of patients with FM. Further studies are needed to validate this sign for diagnosis and follow up of FM patients.

\section{References:}

[1] American Academy of Dermatology. (2007, November 12). Feeling Stressed? How Your Skin, Hair And Nails Can Show It. Science Daily. Retrieved December 30, 2019 from www.sciencedaily.com/ releases/2007/11/071109194053.htm

Disclosure of Interests: None declared

DOI: 10.1136/annrheumdis-2020-eular.5317

\section{$\mathrm{AB} 0957$ \\ IS AQUATIC THERAPY MORE EFFECTIVE THAN LAND- BASED THERAPY IN REDUCING PAIN OF WOMEN WITH FIBROMYALGIA?}

S. Rivas Neira ${ }^{1}$, A. Pasqual Marques ${ }^{2}$, J. Vivas Costa ${ }^{1}{ }^{1}$ Universidade da Coruña, A Coruña, Spain; ${ }^{2}$ Faculdade de Medicina da Universidade de São Paulo, São Paulo, Brazil

Background: Fibromyalgia is a rheumatic disorder characterized by chronic widespread pain often associated with fatigue, unrefreshed sleep and cognitive problems with an increasing prevalence. Aquatic therapy has already been used for managing the symptoms of this syndrome. However, it is not clear whether there is a superiority of aquatic therapy over land-based therapy in improving the symptoms of fibromyalgia patients.

Objectives: Determine the effectiveness of two physiotherapy protocols: aquatic therapy versus land-based therapy, for decreasing pain in women with fibromyalgia Methods: The study protocol was a single-blind randomized controlled trial. Forty women diagnosed with fibromyalgia were randomly assigned into two groups: Aquatic Therapy $(n=20)$ or Land-based Therapy $(n=20)$. Both interventions include 60-min therapy sessions, structured into four sections: Warm-up, Proprioceptive Exercises, Stretching and Relaxation. These sessions were carried out three times a week for three months. The variables analyzed were: pain intensity (Visual Analogue Scale [VAS]), pain threshold (algometer), quality of life (Revised Fibromyalgia Impact Questionnaire [FIQR]), sleep quality (Pittsburgh Sleep Quality Index [PSQI]), fatigue (Multidimensional Fatigue Inventory [MFI]) and physical ability (6-minute Walk Test [6MWT]). Outcome measures were evaluated at baseline, at the end of the 3-month intervention period, and 6-weeks post-treatment. Statistical analysis will be carried out using the SPSS 21.0 program for Windows and a significance level of $p \leq 0.05$ was used for all tests. Results: At the end of intervention period, both therapies were effective in improving pain intensity $(p<0.05)$, pain threshold $(p<0.05)$, quality of life $(p<0.05)$, fatigue $(p<0.05)$ and physical ability $(p<0.05)$. For sleep quality, only the aquatic therapy group experienced a significant improvement $(p=0.033)$. No differences were 